\section{Atypical Symptom of Ehlers-Danlos Syndrome Impeding Diagnosis: Feeling of Spinal Instability}

\section{To the Editor:}

Ehlers-Danlos syndrome (EDS) has a genetic background and is caused by defects in collagen biosynthesis ${ }^{1}$. The main features of EDS are hyperelasticity, fragility of skin, hyperlaxity of the joints, and bleeding diathesis ${ }^{2,3}$. Several types of EDS are recognized, depending on the specific gene mutations and presented phenotypes. In type VI the primary symptom is kyphoscoliosis 4

We describe a patient with EDS with underreported disease related findings that impeded diagnosis: a feeling of spinal instability and balance control disturbances.

A 20-year-old man was admitted to the clinic with spinal instability (Figure 1) and balance control disturbances. The symptoms made normal walking impossible and compromised other daily activities. Because of his symptoms he had been hospitalized several times on pediatric wards with a diagnosis of kyphoscoliosis and Scheuerman syndrome. During the hospitalizations he received psychological care, as no organic causes of the symptoms could be identified.

Physical examination revealed no symptoms of nervous system or

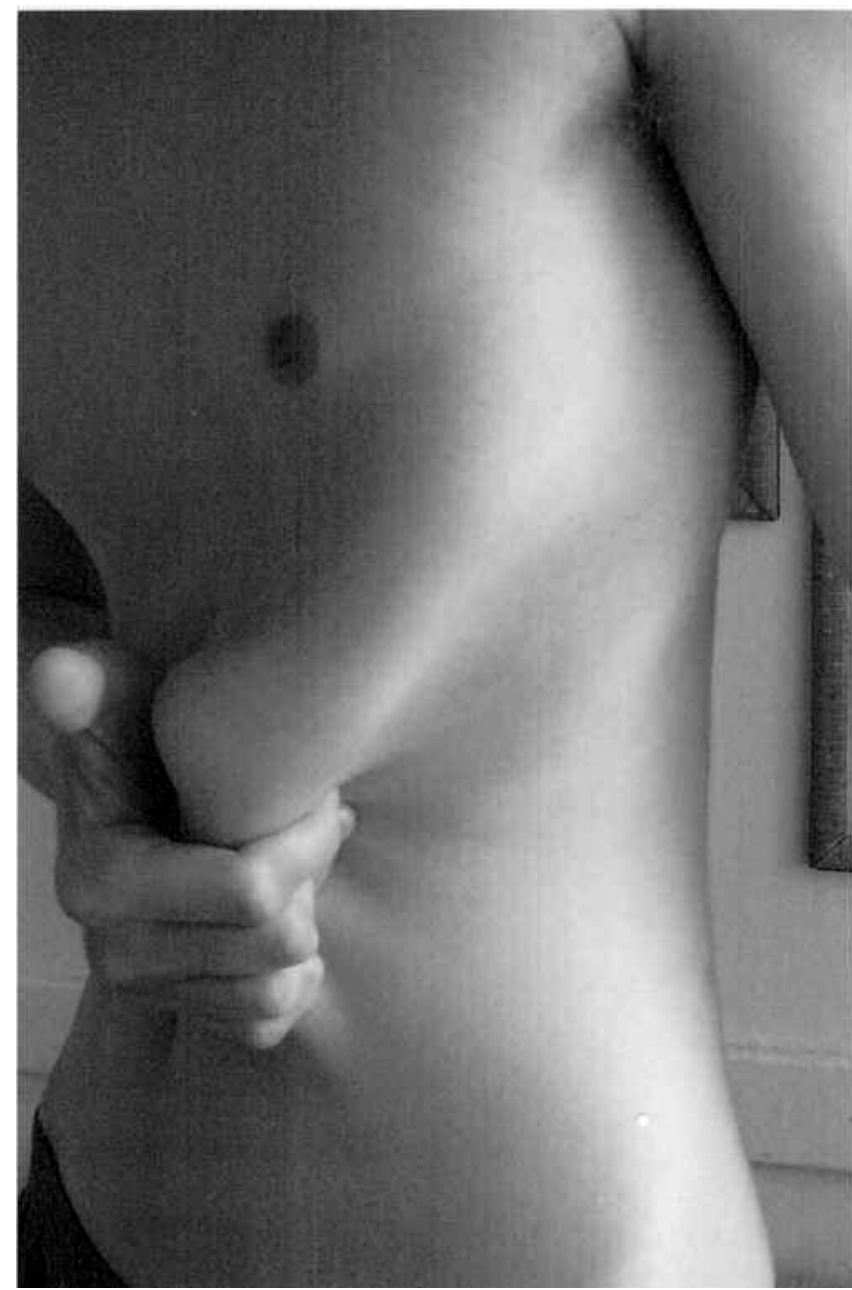

Figure 1. Manipulation, by a patient with EDS, of the costal arch, exceeding the physiologic range of movement and changing the shape of the chest; this is possible due to hypermobility of the costal cartilage connecting ribs 8,9 , and 10 with the sternum, and sternocostal and costotransverse joints. labyrinth damage. Increased range of movement in all joints of the upper extremities, lower extremities, spine and thorax; hyperelasticity of skin; and hypertrophic skin striae on the back were noted (Figure 1,2). An ophthalmologist diagnosed the patient with myopia. Position change from lying to sitting or standing caused increased thoracic hyperkyphosis and cervical hyperlordosis in about 30 seconds. Increasing anteversion of the trunk was accompanied by a seeking of support points and positioning the legs in a wide stance. The patient felt as though he was losing control over his spine and losing his balance. His medical history revealed the presence of kyphoscoliosis in a sister and a cousin. There were no significant abnormalities seen on radiological examination of the spinal canal and brain, biochemical tests or electrophysiological examination. Radiography, computed tomography, and magnetic resonance imaging showed a kyphoscoliotic position of the spine.

Based on the medical history and physical examination he was diagnosed with hyperlaxity of the joints related to $\operatorname{EDS}^{2,3,5}$. Genetic tests confirming type VI EDS were not carried out, as this option was not available.

A feeling of poor joint stabilization is often described in patients with EDS. Our case highlights the feeling of spine instability when assuming a standing position. The feeling was so strong in our patient that it dominated the clinical picture. When studying this case one could assume that the equal contribution of posture coordination disturbances and a balance control disorder hindered diagnosis. In other words, neurological and laryngological causes were searched for to explain poor balance control, ignoring mechanical insufficiency of tissues responsible for spine stabilization and correct posture.

Balance disturbances reported by the patient resulted from gravity-induced changes in spinal curvature caused by low muscle tone and hypermobility in spinal joints ${ }^{6}$. Insufficient muscular stabilization of the spine was indirectly responsible for development of hyperkyphosis. This caused hypermobility of the gravity center, interfering with patient's posture disturbances. The described etiology of posture disturbances is supported by the evolution of the disease. Loading to the spine induced by position change from lying to standing or by standing revealed insufficiency of spinal structures responsible for an upright position. In several dozen seconds the position evolved into thoracic hyperkyphosis, which forced the patient to seek support to avoid a fall. A psychogenic etiology of the described disorders can be excluded based on his repeated cycles of changes in spine curvatures during standing, visibly wide range of thoracic spine curvature during standing and sitting, attempts by the patient to maintain correct posture, and psychological examination. Neurological examination helped to exclude another etiology of posture disturbance, e.g., Parkinson's disease or dystonic disorder ${ }^{7,8}$

Apart from the described case, the biomechanics of poor balance control is often seen in various other motor organ pathologies, e.g., in older people in whom thoracic hyperkyphosis and compromised muscular stabilization increase the risk of falls 9 .

ROBERT GASIK, MD, PhD; TADEUSZ STYCZYNSKI, MD, PhD, Spondylo-Neurosurgery and Neurology Department; and the Pain Clinic, Eleonora Reicher's Rheumatology Institute, Warsaw, Poland. E-mail: robertg@ir.ids.pl

\section{REFERENCES}

1. Walker LC, Overstreet MA, Willing MC, et al. Heterogeneous basis of the type VIB form of Ehlers-Danlos syndrome (EDS VIB) that is unrelated to decreased collagen lysyl hydroxylation. Am J Med Genet A 2004;131:155-62.

2. Beighton P, Price A, Lord J, et al. Variants of the Ehlers-Danlos syndrome. Clinical, biochemical, hematological, and chromosomal features of 100 patients. Ann Rheum Dis 1969;28:228-45.

3. Osborn TG, Lichtenstein JR, Moore TL, Weiss T, Zuckner J. Ehlers-Danlos syndrome presenting as rheumatic manifestation in the child. J Rheumatol 1981;8:79-85. 


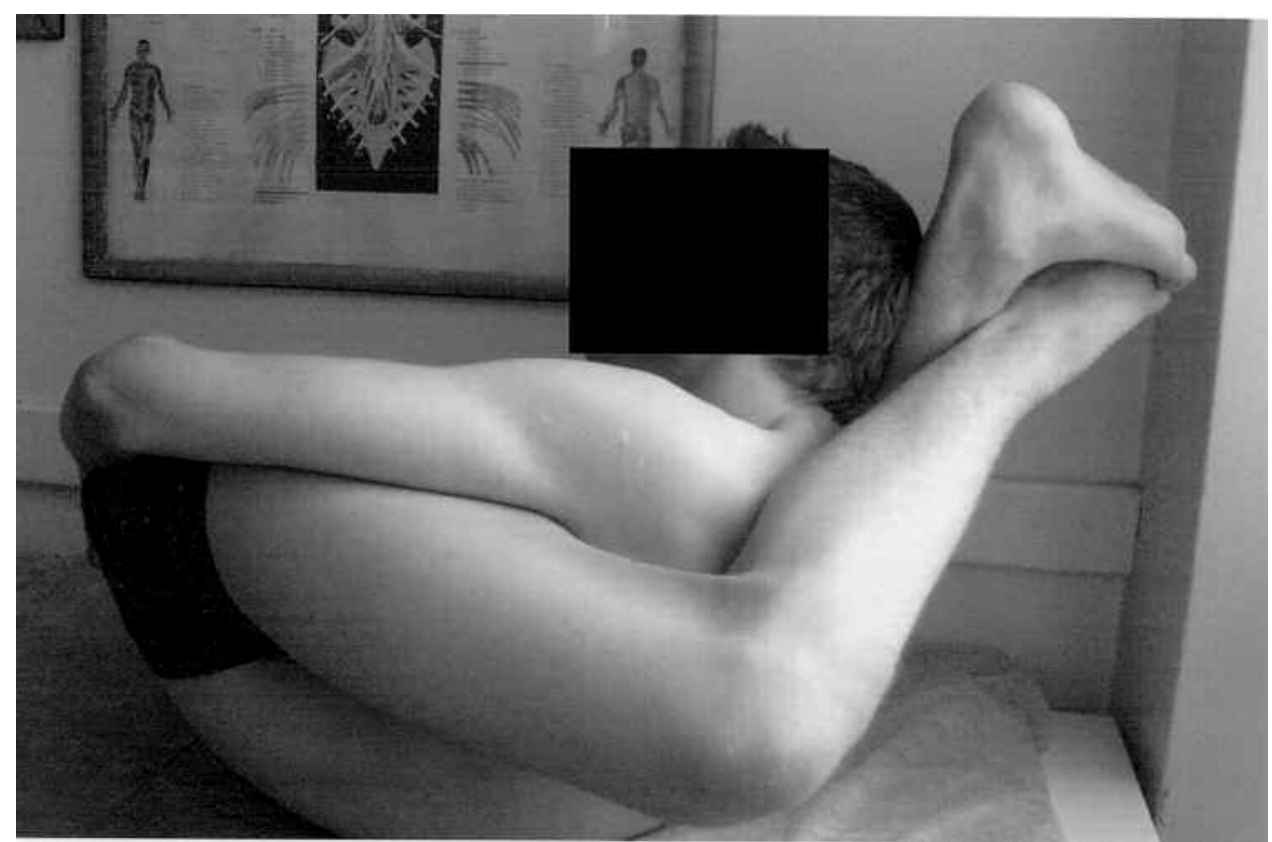

Figure 2. Spinal joint movements demonstrated by a patient with EDS. Note the hyperkyphotic position of the lumbar and thoracic spine.

4. Salavoura K, Valari M, Kolialexi A, Mayrou A, et al. A case of Ehlers Danlos syndrome type VI. Genet Couns 2006;17:291-4.

5. Voermans NC, Engelen BG. Differential diagnosis of muscular hypotonia in infants: The kyphoscoliotic type of Ehlers-Danlos syndrome (EDS VI). Neuromuscul Disord 2008;18:906-7.

6. Bsertin P, Treves R, Julia A, et al. Ehlers-Danlos syndrome, clotting disorders and muscular dystrophy. Ann Rheum Dis 1989;48:953-6.

7. Azher SN, Jankovic J. Camptocormia: Pathogenesis, classification and response to therapy. Neurology 2005;65:355-9.
8. Chastan N, Debono B, Maltête D, et al. Discordance between measured postural instability and absence of clinical symptoms in Parkinson's disease patients in the early stages of the disease. Mov Disord 2008;23:366-72.

9. Kado DM, Huang MH, Nguyen CB, et al. Hyperkyphotic posture and risk of injurious falls in older persons: the Rancho Bernardo Study J Gerontol A Biol Sci Med Sci 2007;62:652-7.

J Rheumatol 2009;36:8; doi:10.3899/jrheum.081146 